\title{
A SIMULATOR STUDY OF DRIVER RESPONSE TO CHANGEABLE MESSAGE SIGNS OF DIFFERING MESSAGE LENGTH AND FORMAT
}

\author{
Jose Guerrier \\ University of Miami School of Medicine \\ Jerry Wachtel \\ The Veridian Group, Inc.
}

Highway congestion nationwide continues to increase, and three Florida urban areas rank among the top ten. Congestion costs the State \$3.5 billion annually in fuel and lost time, and is worsening because of: a) increasing population, b) projected travel increases, and c) recent legislation limiting additional highway capacity.

Florida has been studying and implementing ITS technologies to address its congestion problems, with a focus on its special populations such as the elderly and multi-cultural groups for which English is not the primary language. One of these technologies most widely deployed is the changeable message sign (CMS). Fifty-two CMS are operational in Florida, with 39 more scheduled for deployment soon. Long-range plans will extend this technology Statewide.

Although CMS have the potential to facilitate travel, certain issues must be considered to ensure that they do not exacerbate the congestion problem. Research has resulted in the development of guidelines which address message visibility, legibility and understandability. While these guidelines are helpful in informing the use of CMS, sign operation varies across jurisdictions, often without the basis of empirical support. Because successful CMS operation depends, in part, on driver information processing speed and linguistic ability, there is a need to evaluate CMS with Florida's special populations. This study reports on an effort to determine the importance of specific CMS operational characteristics for drivers in general and elderly drivers in particular.

One key CMS operational issue is the number of phases required to present a complete message. In one Florida jurisdiction about half of all CMS messages require one phase, and half require two. In another jurisdiction, fully 95 percent of CMS messages occupy two phases. There are also known cases of CMS using three-phase messages. "On-time” for two-phase messages varies from 2.5 to 5 seconds per phase across the State. Of course, the appropriateness of this on-time depends not only on the characteristics of the CMS itself (letter size, font, brightness, contrast, etc.), but on road, traffic and weather conditions, and, of course, on driver characteristics such as visual acuity, information processing speed, reading ability, and comprehension of English.

This study, funded by the National Institute on Aging (NIA), investigated issues related to the number of CMS phases and their on-time. We used a low-cost, interactive driving simulator supplemented with a video monitor above the main display. While simulator screens presented interactive road and traffic conditions the supplemental monitor displayed the CMS. Young and old drivers drove the simulator under different workload conditions and responded to road closure/detour information on the CMS. All CMS displays were developed in accordance with accepted guidelines and were reviewed for content by independent experts. 
Results showed consistent and significant age effects across all tested conditions. In addition, we found significantly poorer response for all drivers under the two-phase CMS, despite the fact that our message "on-time” was nearly 2 seconds longer than that used in two major Florida jurisdictions. These findings have implications for CMS design and operation in Florida and in other jurisdictions with similar populations. 